BMJ Open

Diabetes

Research

\& Care

\title{
Recurrence rates suggest delayed identification of plantar ulceration for patients in diabetic foot remission
}

\author{
Brian J Petersen (10 , ${ }^{1}$ Sicco A Bus, ${ }^{2}$ Gary M Rothenberg, ${ }^{3}$ David R Linders, ${ }^{1}$ \\ Lawrence A Lavery, ${ }^{4}$ David G Armstrong ${ }^{5}$
}

To cite: Petersen BJ, Bus SA, Rothenberg GM, et al. Recurrence rates suggest delayed identification of plantar ulceration for patients in diabetic foot remission. BMJ Open Diab Res Care 2020;8:e001697. doi:10.1136/ bmjdrc-2020-001697

Received 17 June 2020 Revised 1 August 2020 Accepted 5 September 2020

A) Check for updates

(C) Author(s) (or their employer(s)) 2020. Re-use permitted under CC BY-NC. No commercial re-use. See rights and permissions. Published by BMJ.

${ }^{1}$ Podimetrics Inc, Somerville, Massachusetts, USA

${ }^{2}$ Department of Rehabilitation Medicine, University of Amsterdam, Amsterdam, Noord-Holland, Netherlands ${ }^{3}$ Department of Internal Medicine, University of Michigan Medical School, Ann Arbor, Michigan, USA

${ }^{4}$ Department of Plastic Surgery, University of Texas Southwestern Medical Center at Dallas, Dallas, Texas, USA ${ }^{5}$ Department of Surgery, USC Keck School of Medicine, Los Angeles, California, USA

Correspondence to Mr Brian J Petersen; authors@podiography.org

\section{ABSTRACT}

Introduction Foot ulcers are a common and costly complication of diabetes, and delays in treatment can result in impaired healing, infection, hospitalization, and lower extremity amputation.

Research design and methods We aimed to determine whether patterns in plantar diabetic foot ulcer (DFU) recurrence coincided with typical intervals between routine preventive care appointments, which would suggest that delays exist between ulcer development and identification. We completed an analysis of existing data from two multicenter studies in 300 total participants. We analyzed unadjusted counts of DFU binned in weekly intervals and defined 'exam periods' as intervals from 2 to 4 weeks, from 6 to 8 weeks, within 1 week of 3 months and within 1 week of 6 months. We tested whether recurrence rates during exam periods were equivalent to rates outside exam periods. We estimated the delay between DFU development and DFU identification such that the rate of development would have been constant.

Results During exam periods, a total of 43 DFUs were identified $(43 / 86=50 \%)$ despite the fact that these periods represent only $23.5 \%$ of follow-up in aggregate. Accounting for censoring, the annualized incidence during exam periods was $0.68 \mathrm{DFU} / \mathrm{year}(\mathrm{Cl} 0.48$ to 0.89 ) in contrast to $0.25 \mathrm{DFU} /$ year (Cl 0.18 to 0.32 ) outside exam periods (incidence ratio $=2.8, \mathrm{Cl} 1.8$ to 4.3 ). We estimated delays between DFU occurrence and identification to average 15.3 days (IQR 7.4-23.7 days).

Conclusions These findings have potential implications for practice, particularly related to the value of telehealth and in-home monitoring of patients in diabetic foot remission. Additionally, there are implications for study design, which should consider the impact of interval censoring and attempt to control for confounders related to frequency and timing of exams.

\section{INTRODUCTION}

Foot ulcers are a common and costly complication of diabetes. ${ }^{1-3}$ A principal determinant of outcomes for those with diabetic foot ulcers (DFUs) is the severity of the wound at presentation. Delays accumulated throughout the care pathways for patients with DFU can result in impaired healing, infection, hospitalization, and lower extremity amputation. ${ }^{45}$ Oyibo and colleagues reported a strong relationship

\section{Significance of this study}

What is already known about this subject?

- Foot ulcers are a common and costly complication of diabetes, and delays in treatment accumulated throughout the care pathways for patients with diabetic foot ulcers (DFUs) can result in impaired healing, infection, hospitalization, and lower extremity amputation.

\section{What are the new findings?}

- In two studies conducted in 300 participants, plantar DFU recurrence appears to be clustered around periods typically associated with routine foot exams. We found the rate of plantar foot ulcer recurrence was a factor of 2.8 greater ( $\mathrm{Cl} 1.8$ to 4.3 ) during short periods coinciding with expected routine exams for those in diabetic foot remission. We estimated delays between plantar foot ulcer occurrence and plantar foot ulcer identification to average 15.3 days (IQR 7.4-23.7 days).

How might these results change the focus of research or clinical practice?

- These findings have potential implications for practice, particularly related to the value of telehealth and in-home monitoring of those at risk of recurrence. Additionally, there are implications for the design of future studies, which should consider interval censoring in their analyses as well as potential confounding factors related to frequency and timing of exams in their statistical comparisons.

between the severity of DFU when identified and subsequent amputation rate. ${ }^{6}$ In 2017, Smith-Strøm and colleagues concluded that 'early detection and referral by both the patient and general practitioner are crucial for optimal foot ulcer healing, ${ }^{7}$

A recent systematic review attributes delays in initiating appropriate treatment to limitations in patient self-care, inaccurate healthcare assessment, and barriers to referral. ${ }^{4}$ Of these, perhaps the most challenging to overcome are those related to the patient, who is often impeded in performing recommended 
daily self-exams and seeking care for emergent DFU by several factors, including comorbid peripheral neuropathy and loss of protective sensation, ${ }^{8}$ comorbid vision impairment, ${ }^{9}$ compromised lower joint mobility, ${ }^{10}$ diminished cognitive function and poor health-related decision-making, ${ }^{11}$ poor recall of diabetic foot education, ${ }^{12}$ social isolation, ${ }^{13}$ and distrust of established clinical pathways due to prior poor healthcare outcomes and experiences. ${ }^{14}$

Thus, despite the importance of timely care for DFU, there are reasons to believe that meaningful delays exist between development of DFU and identification and initiation of treatment by a healthcare provider. Unfortunately, there is little objective evidence related to these delays.

We hypothesized that a disproportionate number of recurrent DFUs are first identified and treated by a provider during scheduled routine exams. We explored whether this hypothesis manifests in temporal patterns in plantar DFU recurrence rates. We analyzed data from 300 patients who participated in two multicenter studies to determine if patterns in plantar DFU recurrence rates existed and if these patterns coincided with expected intervals between routine preventive care exams. We estimated the average delay between plantar DFU occurrence and identification of the DFU by the provider by imposing that the rate of recurrence should be constant.

\section{RESEARCH DESIGN AND METHODS}

\section{Data provenance}

We used existing data from two longitudinal prospective studies, one of which was a multicenter study conducted in 171 participants in the Netherlands and the other a multicenter study conducted in 129 participants in the USA. These studies were selected because their data were the only data appropriate and available to the authors at the time of the analysis. We declare no competing interests with respect to the selection of these studies for inclusion in this analysis.

The first study, Bus et $a l^{15}$ (Dutch Trial Register NTR1091), was undertaken at two academic hospitals and eight large general hospitals in the Netherlands between 2007 and 2010. The investigators enrolled 171 participants who had healed from a plantar DFU in the 18 months prior to enrollment. The study was designed to assess the impact of custom pressure-improved footwear and adherence to use on plantar DFU recurrence. Participants were randomized into two arms. One arm received custom-made footwear without modification based on pressure measurement, and the other received customized footwear which was modified using pressure data. Because a non-significant difference was found at 18 months in ulcer-free survival between the two arms, both have been included here.

Participants were required to be older than 18 years of age, have type 1 or type 2 diabetes mellitus, documented loss of protective sensation due to peripheral neuropathy, and a plantar DFU that had healed within the 18 months prior to study enrollment. Patients were excluded from participation if they had bilateral amputation more proximal than the tarsometatarsal joint, required the use of walking aids that provided an offloading effect, or had severe illness which in the judgment of the investigator would make survival for 18 months unlikely.

On enrollment, the investigators conducted a physical exam and collected a detailed medical history. Participants were evaluated every 3 months at their hospitals and otherwise received routine care with specialist providers (mainly podiatrists) and diabetes-educated pedicurists per standard care. Of all planned 3-month follow-up visits, $97 \%$ occurred. A panel of blinded diabetic foot experts diagnosed any lesions suspected of being a DFU based on digital photos.

The primary outcome was survival from plantar DFU recurrence over the 18 months of follow-up, with last observation carried forward for the six participants who died, and analysis of the medical records at 18 months for those participants who dropped out.

The second study, Frykberg et $a l^{16}$ (ClinicalTrials. gov NCT02647346), was undertaken at seven enrolling sites in the USA between 2013 and 2016. The investigators enrolled 129 participants and followed each for 34 weeks under standard preventive foot care plus a daily-use remote temperature monitoring mat. This study was designed to assess the accuracy of once-daily foot temperature monitoring, and therefore providers and participants remained blinded to the thermometric data, rendering the device a sham. The authors of the present study hypothesize that the sham did not impact the observed rate of recurrence.

Participants were required to have documented history of diabetes and healed plantar DFU, be ambulatory, have ankle brachial index exceeding 0.5 , and be at least 18 years of age at the time of enrollment. Exclusion criteria were having an unhealed plantar DFU, history of amputation more proximal than Chopart disarticulation, active Charcot arthropathy, end-stage renal disease, immunosuppressive disease, cognitive deficit preventing the participant from providing informed consent, and any other factor deemed disqualifying by the investigators.

On enrollment of each participant, the investigators conducted a physical exam and collected a detailed medical history. Participants were instructed to notify the study staff if they noticed new lesions or DFU during participation, at which point the investigators scheduled an appointment to triage and treat the reported lesions per standard care. In addition to providing routine diabetic foot exams in accordance with standard practice, study staff also contacted each participant by phone during months 2 and 4 of participation to inquire whether the participant had noticed any changes in the feet during daily self-exam, including query regarding development of DFU and associated timing. Finally, each participant was examined in clinic at the completion of the 34-week study, and a final chart review was completed 
to ensure all preulcerative and ulcerative lesions during participation were documented as study outcomes.

The study's primary outcome of interest was plantar DFU recurrence. The investigators characterized the severity and location of all DFU identified during the 34-week follow-up or until the participant withdrew consent, although the present analysis considers only the first recurrent DFU for each participant.

In both trials, each outcome was associated with the earliest date for which there was documentation from a healthcare professional that a DFU was present and could be evaluated and treated.

\section{Analysis plan}

We calculated the distribution of intervals between consecutively identified DFU over the first 34 weeks from enrollment in both trials, representing the follow-up period for Frykberg et $a l,{ }^{16}$ which was the shorter of the two trials considered. We tested whether the interval distribution was exponential, as would be expected if DFU recurrence was Poisson distributed with constant rate. We used a one sample Kolmogorov-Smirnov test with $\alpha=0.05$.

We analyzed unadjusted counts of DFU binned in weekly intervals. We defined 'exam periods' to be from 2 to 4 weeks, from 6 to 8 weeks, within 1 week of 3 months, and within 1 week of 6 months. These periods were based on the clinical experience of the authors and were chosen to coincide with typical follow-up intervals between wound healing and routine examinations in the care environments studied. As is noted in the Prevention Guidelines from the International Working Group on the Diabetic Foot, 'screening frequency is based on expert opinion, since there is no published evidence to support these intervals'. ${ }^{, 7}$

We modeled recurrence during the exam periods as a homogeneous Poisson process, a common epidemiological model that assumes observations are generated by a Poisson distribution with constant rate. ${ }^{18}$ We modeled recurrence outside exam periods as another homogeneous Poisson process and compared the two processes for equivalence at a significance of $\alpha=0.05$. We also computed the incidence ratio (IR) and associated CIs from the Poisson processes. We reported unadjusted data from each study to understand qualitatively whether any trends or patterns in recurrence were common to each.

We then considered the impact of censoring. We compared the Kaplan-Meier curves between the two studies using Wellek's log-rank test for survival equivalence at a significance of $\alpha=0.05 .{ }^{19}$ We constructed a Nelson-Aalen estimator for the cumulative hazard of the combined trials over the 34 weeks for which both studies had participants enrolled. ${ }^{20}$ To estimate the hazard rate of recurrence, which can be interpreted as the instantaneous rate of recurrence, we differentiated the cumulative hazard using an Epanechnikov kernel with a bandwidth resolution of 1 week, which is optimal in a mean square error sense. ${ }^{21}$
We acknowledge that the etiology for plantar DFU is multifactorial and most commonly includes neuropathy, foot deformity and acute or repetitive trauma. Given these varied risk factors and in an attempt to assess the average delay between DFU occurrence and identification of the DFU by the provider, we assumed that there ought to be a constant rate of recurrence during the trial and that any clustering in identification of DFU is due to delays between development of DFU and identification during routine exams. Under this assumption, we solved for the delay for each participant who was identified to have a DFU by minimizing the time between assumed ulcer recurrence. We reported summary statistics for this distribution of delays between development and identification.

\section{RESULTS}

A total of 103 of the 300 participants $(34.3 \%)$ developed recurrent plantar DFU across both study follow-up periods. During the 34 weeks of follow-up in which both studies had participants enrolled, 86 participants developed a DFU (28.7\%). Descriptive statistics for these DFUs are available in the original publications. ${ }^{1516}$

Figure 1A shows the distribution of intervals between consecutively identified DFU. We found that the observed distribution of intervals between successive DFU was not exponentially distributed $(\mathrm{p}<0.01)$, implying that recurrence of plantar DFU indexed from enrollment is not Poisson distributed with constant rate. This is confirmed qualitatively in the qq plot in figure $1 \mathrm{~B}$, which shows deviations from the exponential distribution, particularly in the higher quartiles.

Figure 2 shows the unadjusted counts of DFU binned in weekly intervals over the first 34 weeks from enrollment in both trials. During the exam periods, a total of 43 plantar DFU were identified $(43 / 86=50 \%)$ despite the fact that these periods represent only $23.5 \%$ of the whole follow-up period in aggregate (8 weeks of 34 total weeks). The annualized incidence of recurrence in the former is $0.68 \mathrm{DFU} /$ year (CI 0.48 to 0.89 ) and $0.25 \mathrm{DFU} /$ year (CI 0.18 to 0.32 ) in the latter. These rates imply an IR of 2.8 (CI 1.8 to 4.3 ).

Table 1 shows the breakdown by interval and in each study. The rates of recurrence during each period are qualitatively similar across the two studies, although a larger proportion of participants from Frykberg et $a l^{16}$ were found with recurrence between weeks 2 and 4 , and a larger percentage of participants in Bus et $a l^{15}$ were identified at approximately 3 months, as might be expected, given the required 3-month follow-up in this trial. The IR for the exam period in each trial was also similar (2.6 in Frykberg $e t a l^{16}$ vs 2.9 in Bus $e t a l^{15}$ ).

Figure 3 shows the DFU-free survival distributions for each of the two trials. These distributions were found to be statistically equivalent $(\mathrm{p}<0.01)$. Approximately $30 \%$ of participants were reported to have a DFU within 34 weeks of enrollment in the two studies. 

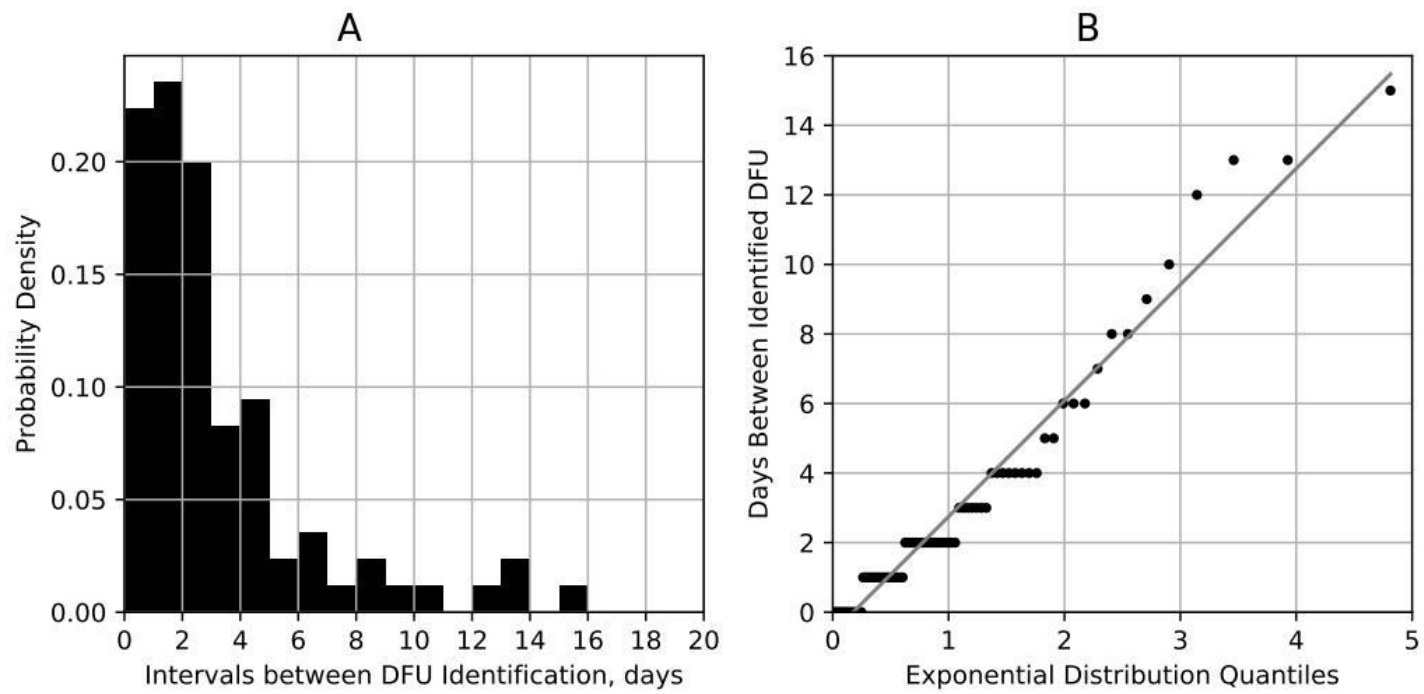

Figure 1 (A) Distribution of intervals between DFU being identified in the two trials ${ }^{15}{ }^{16}$; (B) qq plot of these intervals assuming Poisson-distributed incidence. DFU, diabetic foot ulcer.

Figure 4 shows the recurrence hazard rate (rate of recurrence as a function of time) for plantar DFU identified over both trials. Distinct peaks in DFU recurrence exist between weeks 2 and 4, weeks 6 and 8, and approximately centered on month three and month six. These peaks correspond to clusters of DFU identified during these periods, as demonstrated in the third panel of the figure. A total of 104 participants $(34.7 \%)$ were censored at the end of 34 weeks (either due to identification of DFU, dying, or dropping out of the study).

We estimated delays between DFU occurrence and DFU identification to be, on average, greater than 2 weeks (15.3 days, IQR 7.4-23.7 days). The 95th percentile of these delays was 31 days.

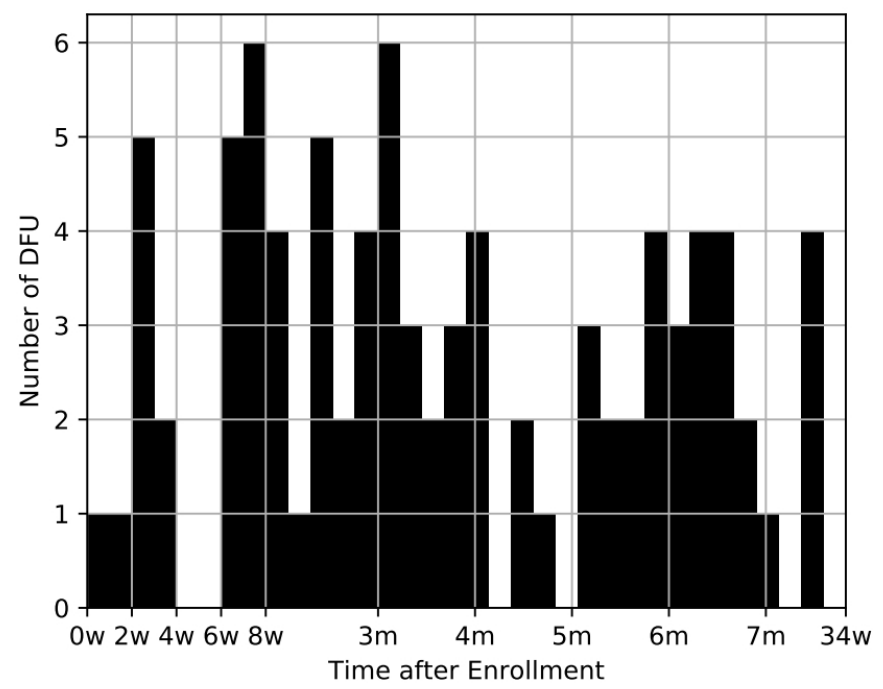

Figure 2 Unadjusted counts of DFU binned into weekly intervals from enrollment. DFU, diabetic foot ulcer.

\section{DISCUSSION}

We believe our study to be the first to report rates of plantar DFU recurrence as a function of time after an index exam, in this case corresponding to enrollment in one of two multicenter trials. We analyzed data from 300 participants in these two trials and found that recurrence rates were not constant and recurrence was not Poissondistributed over the 34 weeks of follow-up during which both studies had participants enrolled $(\mathrm{p}<0.01)$. Instead, recurrence appears to be clustered around periods typically associated with routine exams in diabetic foot patients. Ignoring participant censoring, we reported that $50 \%$ of plantar DFU were identified during periods that represented only $23 \%$ of participant follow-up. Adjusting for censoring, we found the rate of recurrence was a factor of 2.8 greater during periods typically associated with routine exams for those in diabetic foot remission. Qualitatively, the findings were consistent across the two studies.

Our study may also be the first to estimate delays between development and identification of plantar DFU independently of subjective reports by participants. By assuming that rates of recurrence should be constant over 34 weeks, we estimated these delays to average 15.3 days (IQR 7.4-23.7 days). These results are possibly consistent with and help explain data from Frykberg et al, ${ }^{16}$ which reported that inflammation (manifesting as temperature differences between contralaterally matched locations on the feet) precedes identification of DFU by 35 days on average. These investigators have recently reported that in-home monitoring of diabetic foot complications may be useful not only for the prevention of DFU, as several previous studies have shown, ${ }^{22-24}$ but also for their early detection. ${ }^{25} 26$ This observation also suggests delayed identification of DFU and is potentially consistent with our findings in the present study. 
Table 1 Distribution of DFU identification by time after enrollment and study

\begin{tabular}{lccc}
\hline & Bus et al $^{\mathbf{1 5}}$ & Frykberg et al $^{\mathbf{1 6}}$ & Total \\
\hline All DFU & 49 & 37 & 86 \\
$2-4$ weeks & $3(6.1 \%)$ & $4(10.8 \%)$ & $7(8.1 \%)$ \\
68 weeks & $7(14.3 \%)$ & $4(10.8 \%)$ & $11(12.8 \%)$ \\
3 months \pm 1 week & $8(16.3 \%)$ & $4(10.8 \%)$ & $12(14.0 \%)$ \\
6 months \pm 1 week & $7(14.3 \%)$ & $6(16.2 \%)$ & $13(15.1 \%)$ \\
Remainder of study & $24(49.0 \%)$ & $19(51.4 \%)$ & $43(50.0 \%)$ \\
\hline
\end{tabular}

DFU, diabetic foot ulcer.

One strength of our study and approach is that we are able to estimate delays between development of plantar DFU and initiation of treatment without relying on participant subjective histories, which are potentially unreliable due to comorbid peripheral neuropathy, vision impairment, and limited joint mobility. Another strength of our effort is that we relied on two studies conducted in different care environments, by different investigators, and with different outcomes of interest. Despite these differences, both studies showed similar patterns of recurrence, with a disproportionate number of DFUs being identified during periods typically associated with routine follow-up exams.

An important weakness of our study is that we have made a strong assumption: that rates of recurrence ought to be constant over at least 34 weeks of follow-up when indexed from the exam at enrollment. Instead, it is possible that the clustering of DFU observed in both studies is due to a progressive physiological process which is 'renewed' at each clinical exam. For example, many

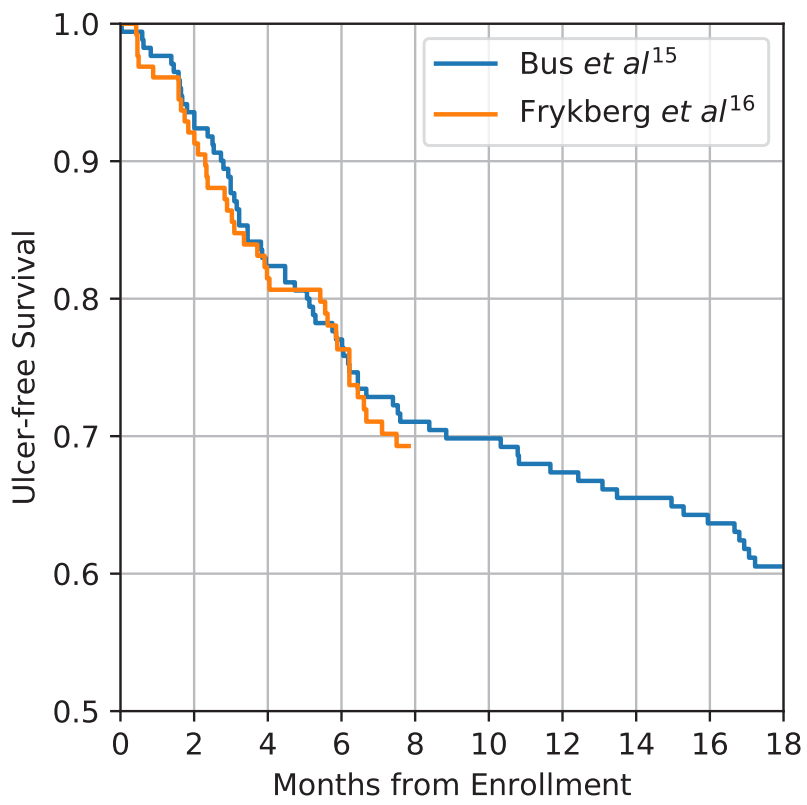

Figure 3 Diabetic foot ulcer-free survival comparison between Bus et $a l^{15}$ and Frykberg et $a l^{16}$ indexed from enrollment. patients with diabetic foot syndrome develop hyperkeratotic lesions progressively over time, and these lesions are known to impart risk for DFU recurrence. ${ }^{27} 28$ In fact, many routine podiatric visits are scheduled precisely for debridement of these lesions. ${ }^{17}$ Were routine exams well timed to coincide with the development of DFU due to increased pressure and repetitive microtrauma from
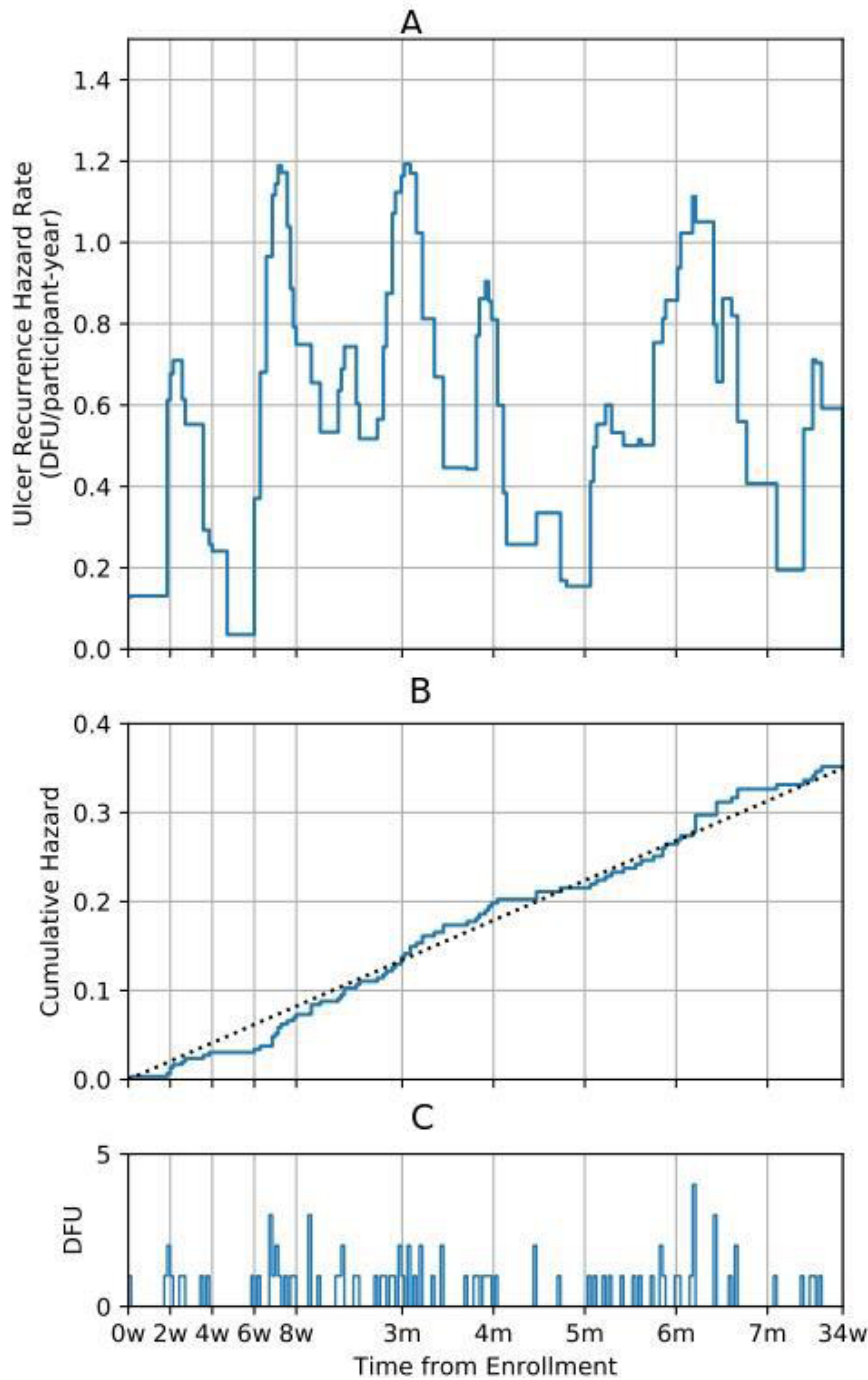

Figure 4 (A) Hazard rate of DFU identification, (B) cumulative hazard of DFU identification over the two studies, and (C) number of DFU identified. DFU, diabetic foot ulcer. 
callus, this alternative hypothesis could explain our findings in part or whole and would invalidate the delays between plantar DFU development and identification that we have calculated. However, were this alternative hypothesis to be true, it would also suggest that more frequent treatment of hyperkeratotic lesions is needed to avoid DFU development secondary to callus, and the delays we have reported may better coincide with the initial development of callus and its progression until routine exam. Under this alternative hypothesis, a principal goal of care for managing populations in diabetic foot remission may be to eliminate clusters of recurrence, and the methods presented here may prove useful for assessing and monitoring quality of care for those with a history of plantar DFU.

Another weakness of our effort is that we had to assume typical intervals for scheduled routine exams because neither study characterized the timing and nature of the exams that had occurred during follow-up (eg, we did know whether an exam was scheduled for routine care as opposed to scheduled for an emergent issue such as a participant developing a DFU). While the exam periods we observed match clinical practice in our experience for the care environments in which the studies were performed, or to coincide with exams or telephone interactions obligated by the study protocol (eg, at 2 and 4 months for Frykberg et $a l^{16}$ and in 3-month intervals for Bus et $a l^{15}$ ), it is possible that the elevated incidence during our defined exam periods actually did not coincide with true scheduled routine exams.

Future studies attempting to confirm our findings prospectively should characterize the dates of all foot exams. Investigators should also distinguish between those wounds that were identified by participants who subsequently scheduled an exam for evaluation and treatment and those which were found incidentally during routine exams scheduled by the provider. Furthermore, investigators should characterize whether the DFU presented with hyperkeratotic covering to explore whether clustering of wounds may be related to the progressive nature of hyperkeratosis as opposed to delays in identification. For this same reason, future studies should capture and report the frequency and thickness of callus debrided for exams during which a DFU was not identified. A properly designed prospective study for our aim may also choose to collect subjective history from participants when a DFU is identified to assess whether the participant was aware of the wound and, if so, for how long. Although such data should not be considered reliable, it can be potentially used as confirmatory evidence or to set a lower bound on inferred delays between DFU development and identification.

Our findings have several potential implications and raise important questions for clinical practice. We have quantified the delays impeding prompt care of DFU, which may challenge today's standard of care for management of high-risk populations. To reduce potential delays between DFU development and identifications, we must address factors related to both patients and the healthcare system.

The first question prompted by our findings is when and by whom are DFU found? As previously mentioned, several authors have characterized limitations, making daily self-examinations difficult for patients living with diabetes. Caregivers can certainly assist in daily foot exams, potentially resulting in earlier identification and better outcomes. Additionally, those patients with caregiver support may be more likely to seek medical attention sooner.

Next, if a patient or caregiver discovers a concerning clinical finding such as signs of infection or open wound with drainage, what action is taken? Patients often experience apathy and rationalize delays in seeking care. ${ }^{29}$ Furthermore, for patients who do seek care identifying a potential diabetic foot complication at home, the patient may experience issues related to access to needed healthcare, such as provider unavailability or scheduling conflicts causing further delays in treatment.

Third, what are the clinical implications of a significant delay in treatment, and how are appropriate intervals for preventative care established? Whether intentional or unintentional, delays in prompt care for DFU can lead to more devastating and costly outcomes. In this study, our findings suggest that there may exist an average delay of 2 weeks prior to identification, documentation, and treatment of a recurrent plantar DFU. Several risk stratification systems and clinical practice guidelines exist, and the International Working Group on the Diabetic Foot has suggested intervals for routine care visits with a foot care specialist. ${ }^{17}$ However, there appears to be little scientific basis for these recommendations. ${ }^{30}$ Furthermore, scarce evidence-based guidance exists for personalization of routine follow-up individual patients based on specific risk factors. ${ }^{31}$ Care intervals should likely be individualized based on the risk factor spectrum, activity level, and patient history. Better evidence in this area may help improve the timing and cadence of routine preventive care by a specialist provider, with the principal goal of reducing recurrence.

Finally, what can be done to minimize delays between plantar DFU development and identification by providers? Patients deemed at high risk of recurrence should be educated on the urgency and seriousness of an impending problem and seek immediate attention. Additionally, impediments to access to care must be addressed. Advances in home monitoring and telemedicine can also play a role in plantar DFU recurrence prevention. In two recent publications, Najafi and colleagues explored the goal of increasing ulcer-free days through the use of remote monitoring devices, wearable technologies, and mobile health technologies. ${ }^{32} 33$ They suggest that the novel use of technology can help identify potential problems and triage patients for timely interventions. Many of these technologies can be considered an extension of telemedicine options or enable 'eyes on the patient' in the home to facilitate needed clinical interventions with 
minimal delays. We believe that, with advances made in home-based monitoring and telehealth in the peripandemic period, significant improvements in detection and reporting may be possible..$^{34-36}$

If true, our findings would also have important potential implications for the analysis and interpretation of data from studies with diabetic foot recurrence as an outcome. The results suggest that identification of a plantar DFU by investigators during a trial may not be a good indication of when the DFU actually occurred due to delays in identification until routine or scheduled exams. Thus, it is likely inappropriate to assume DFU-free survival through the date that a DFU was identified by the investigator, which is a typical assumption underlying conventional survival analysis as applied in the diabetic foot literature. In reality, if our findings are confirmed, actual survival durations to plantar DFU development are over-reported due to delays in identification.

Conventional survival analysis naturally accommodates right censoring, where the outcome of interest was not observed and survival is known to be longer than a certain duration; however, when the outcome is known to occur between two dates (eg, dates of successive follow-up exam, the first on which the investigator does not identify a DFU and the second on which the investigator does), this is appropriately modeled as interval censoring, not right censoring. Interval censoring is generally much less informative than right censoring and results in lower power to detect differences between survival distributions. ${ }^{37}$ Special methods have been devised to handle this type of censoring and should be considered by researchers designing trials with diabetic foot survival as a primary outcome of interest. ${ }^{38}$ Additionally, the actual frequency and intervals between office visits ought to be a variable reported and controlled for between groups in well-designed controlled trials because differences in frequency of exam can result in bias for interval-censored outcomes.

In conclusion, we have presented evidence that recurrence of plantar DFU is clustered around short periods coinciding with typical timing of scheduled routine exams for those in diabetic foot remission. Further, we have reported that there may exist delays averaging approximately 2 weeks between development of a DFU and identification and treatment of the DFU by providers. These findings have important implications for clinical practice and research design and analysis, and confirmation through properly designed prospective studies is warranted.

Acknowledgements Abbie R. Liu contributed to the copyediting and assembly of this manuscript.

Contributors BJP and DRL: concept and design, analysis of data, interpretation of data and preparation of the manuscript. SAB: concept and design, collection of data, interpretation of data and preparation of the manuscript. GMR: interpretation of data, collection of data, preparation of the manuscript. LAL and DGA: concept and design, interpretation of data and preparation of the manuscript. All authors contributed to critically revise the manuscript and have given final approval of the version to be published.
Funding There is no funding to report for the secondary analysis of existing data. Funding details for the two studies on which this research is based are cited in the original publications.

Competing interests BJP and DRL are employees and shareholders of Podimetrics, Inc. GMR is a consulting medical director for Podimetrics Inc. LAL and DGA are on the scientific advisory board of Podimetrics Inc. SAB reports no competing interests.

\section{Patient consent for publication Not required.}

Ethics approval This study is a secondary analysis of existing deidentified data from two trials which received ethics approval. Informed consent for these trials allowed use of data collected for research purposes, including secondary analyses. Ethics approval details are cited in the original publications for these two studies. The first study, Bus et al, was approved by the medical ethics committees of all 10 participating centers. All participants gave informed consent. The second study, Frykberg et al, was approved by the New England Institutional Review Board and local review boards as required by each site. All participants gave informed consent.

Provenance and peer review Not commissioned; externally peer reviewed.

Data availability statement Data and code are available for research purposes upon reasonable request to the corresponding author.

Open access This is an open access article distributed in accordance with the Creative Commons Attribution Non Commercial (CC BY-NC 4.0) license, which permits others to distribute, remix, adapt, build upon this work non-commercially, and license their derivative works on different terms, provided the original work is properly cited, appropriate credit is given, any changes made indicated, and the use is non-commercial. See: http://creativecommons.org/licenses/by-nc/4.0/.

ORCID iD

Brian J Petersen http://orcid.org/0000-0002-9552-5859

\section{REFERENCES}

1 Armstrong DG, Boulton AJM, Bus SA. Diabetic foot ulcers and their recurrence. N Engl J Med 2017;376:2367-75.

2 Zhang Y, Lazzarini PA, McPhail SM, et al. Global disability burdens of diabetes-related lower-extremity complications in 1990 and 2016. Diabetes Care 2020;43:964-74.

3 Armstrong DG, Swerdlow MA, Armstrong AA, et al. Five year mortality and direct costs of care for people with diabetic foot complications are comparable to cancer. J Foot Ankle Res 2020;13:16.

4 Nickinson ATO, Bridgwood B, Houghton JSM, et al. A systematic review investigating the identification, causes, and outcomes of delays in the management of chronic limb-threatening ischemia and diabetic foot ulceration. $J$ Vasc Surg 2020;71:669-81.

5 Armstrong DG, Lavery LA, Harkless LB. Who is at risk for diabetic foot ulceration? Clin Podiatr Med Surg 1998;15:11-19.

6 Oyibo SO, Jude EB, Tarawneh I, et al. The effects of ulcer size and site, patient's age, sex and type and duration of diabetes on the outcome of diabetic foot ulcers. Diabet Med 2001;18:133-8.

7 Smith-Strøm H, Igland J, Østbye T, et al. The effect of telemedicine follow-up care on diabetes-related foot ulcers: a cluster-randomized controlled Noninferiority trial. Diabetes Care 2018;41:96-103.

8 Monami M, Longo R, Desideri CM, et al. The diabetic person beyond a foot ulcer: healing, recurrence, and depressive symptoms. J Am Podiatr Med Assoc 2008;98:130-6.

9 Bruun C, Siersma V, Guassora AD, et al. Amputations and foot ulcers in patients newly diagnosed with type 2 diabetes mellitus and observed for 19 years. The role of age, gender and co-morbidity. Diabet Med 2013;30:964-72.

10 Zimny S, Schatz H, Pfohl M. The role of limited joint mobility in diabetic patients with an at-risk foot. Diabetes Care 2004;27:942-6.

11 Natovich R, Kushnir T, Harman-Boehm I, et al. Cognitive dysfunction: part and Parcel of the diabetic foot. Diabetes Care 2016;39:1202-7.

12 Yuncken J, Williams CM, Stolwyk RJ, et al. People with diabetes do not learn and recall their diabetes foot education: a cohort study. Endocrine 2018;62:250-8.

13 Palaya J, Pearson S, Nash T. Perception of social support in individuals living with a diabetic foot: a qualitative study. Diabetes Res Clin Pract 2018;146:267-77.

14 Albargawi M, Snethen J, Al Gannass A, et al. Relationship between person's health beliefs and diabetes self-care management regimen. J Vasc Nurs 2017;35:187-92. 
15 Bus SA, Waaijman R, Arts M, et al. Effect of custom-made footwear on foot ulcer recurrence in diabetes: a multicenter randomized controlled trial. Diabetes Care 2013;36:4109-16.

16 Frykberg RG, Gordon IL, Reyzelman AM, et al. Feasibility and efficacy of a smart mat technology to predict development of diabetic plantar ulcers. Diabetes Care 2017;40:973-80.

17 Bus SA, Lavery LA, Monteiro-Soares M, et al. Guidelines on the prevention of foot ulcers in persons with diabetes (IWGDF 2019 update). Diabetes Metab Res Rev 2020;36 Suppl 1:e3269.

18 Frome EL. The analysis of rates using poisson regression models. Biometrics 1983;39:665-74.

19 Wellek S. A log-rank test for equivalence of two survivor functions. Biometrics 1993;49:877-81.

20 Collett D. Modelling Survival Data in Medical Research. Chapman and Hall/CRC, 2015

21 Samiuddin M, El-Sayyad GM. On nonparametric kernel density estimates. Biometrika 1990;77:865-74.

22 Lavery LA, Higgins KR, Lanctot DR, et al. Home monitoring of foot skin temperatures to prevent ulceration. Diabetes Care 2004;27:2642-7.

23 Lavery LA, Higgins KR, Lanctot DR, et al. Preventing diabetic foot ulcer recurrence in high-risk patients: use of temperature monitoring as a self-assessment tool. Diabetes Care 2007;30:14-20.

24 Armstrong DG, Holtz-Neiderer K, Wendel C, et al. Skin temperature monitoring reduces the risk for diabetic foot ulceration in high-risk patients. Am J Med 2007;120:1042-6.

25 Banks JL, Petersen BJ, Rothenberg GM, et al. Use of a remote temperature monitoring mat for the early identification of foot ulcers. Wounds 2020;32:44-9.

26 Rothenberg GM, Page J, Stuck R, et al. Remote temperature monitoring of the diabetic foot: from research to practice. Fed Pract 2020;37:114-24.

27 Pavicic T, Korting HC. Xerosis and callus formation as a key to the diabetic foot syndrome: dermatologic view of the problem and its management. J Dtsch Dermatol Ges 2006;4:935-41.

28 Boyko EJ, Ahroni JH, Cohen V, et al. Prediction of diabetic foot ulcer occurrence using commonly available clinical information: the Seattle diabetic foot study. Diabetes Care 2006;29:1202-7.
29 Beattie AM, Campbell R, Vedhara K. 'What ever I do it's a lost cause.' the emotional and behavioural experiences of individuals who are ulcer free living with the threat of developing further diabetic foot ulcers: a qualitative interview study. Health Expect 2014:17:429-39.

30 Netten JJ, Raspovic A, Lavery LA, et al. Prevention of foot ulcers in the at-risk patient with diabetes: a systematic review. Diabetes Metab Res Rev 2020;36:CD007610.

31 Van Netten JJ, Woodburn J, Bus SA. The future for diabetic foot ulcer prevention: a paradigm shift from stratified healthcare towards personalized medicine. Diabetes Metab Res Rev 2020;36 Supp $1: e 3234$.

32 Najafi B, Reeves ND, Armstrong DG. Leveraging smart technologies to improve the management of diabetic foot ulcers and extend ulcer-free days in remission. Diabetes Metab Res Rev 2020;36 Suppl 1:e3239.

33 Basatneh R, Najafi B, Armstrong DG. Health sensors, smart home devices, and the Internet of medical things: an opportunity for dramatic improvement in care for the lower extremity complications of diabetes. J Diabetes Sci Technol 2018;12:577-86

34 Shin L, Bowling FL, Armstrong DG, et al. Saving the diabetic foot during the COVID-19 pandemic: a tale of two cities. Diabetes Care 2020;43:1704-9.

35 Rogers LC, Lavery LA, Joseph WS, et al. All feet on Deck-The role of podiatry during the COVID-19 pandemic: preventing hospitalizations in an overburdened healthcare system, reducing amputation and death in people with diabetes. J Am Podiatr Med Assoc 2020:0000.

36 Rogers LC, Armstrong DG, Capotorto J, et al. Wound center without walls: the new model of providing care during the COVID-19 pandemic. Wounds 2020. [Epub ahead of print: 24 Apr 2020].

37 Zhang Z, Sun J. Interval censoring. Stat Methods Med Res 2010;19:53-70.

38 Rodrigues AS, Calsavara VF, Silva FIB, et al. Use of intervalcensored survival data as an alternative to Kaplan-Meier survival curves: studies of oral lesion occurrence in liver transplants and cancer recurrence. Applied Cancer Research 2018;38:16. 